\title{
A simple non-invasive method for measuring gross brain size in small live fish with semi-transparent heads
}

This paper describes a non-invasive method for estimating gross brain size in small fish with semi-transparent heads, using system camera equipment. Macro-photographs were taken from above on backlit free-swimming fish undergoing light anaesthesia. From the photographs, the width of the optic tectum was measured. This measure (OT-measure) correlates well with the width of the optic tectum as measured from out-dissected brains in both brown trout fry and zebrafish (Pearson $r>0.90$ ). The OT-measure also correlates well with overall brain wet weight in brown trout fry (Pearson $r=0.90)$, but less well for zebrafish $(r$ $=0.79)$. A non-invasive measure makes it possible to quickly assess brain size from a large number of individuals, as well as repeatedly measuring brain size of live individuals allowing calculation of brain growth. 
1 A simple non-invasive method for measuring gross brain size in

\section{small live fish with semi-transparent heads}

3

Joacim Näslund

4 University of Gothenburg, Department of Biological and Environmental Sciences,

5

Box 463, 40530 Gothenburg, Sweden

6

E-mail: joacim.naslund@bioenv.gu.se

7

Telephone: +46317863696 


\section{INTRODUCTION}

9 Many recent studies on fish have investigated gross size of the brain and various brain areas

10 from ontogenetic or evolutionary perspectives (Ebbesson \& Braithwaite, 2012; Gonda, Herczeg

$11 \&$ Merilä, 2013). The brain measurements have been taken using several different methods, e.g.

12 dry or wet mass (Mayer et al., 2010; Devlin et al., 2012), one-dimensional linear measurements

13 (Marchetti \& Nevitt, 2003; Park \& Bell, 2010), two-dimensional dorsal brain areas (Burns \&

14 Rodd, 2008; Park, Chase \& Bell, 2012), ellipsoid volume approximations based on linear

15 measurements (Pollen et al., 2007; Fraser et al., 2012), histological sectioning (Kotrschal \&

16 Palzenberger, 1992; Kihslinger, Lema \& Nevitt, 2006), geometric morphometric analyses (Park

$17 \&$ Bell, 2010; Park, Chase \& Bell, 2012), or magnetic resonance imaging (Ullmann, Cowin \&

18 Collin, 2010; Ullmann et al, 2010). All of these methods require substantial and time consuming

19 preparation of the brains, and the fish need to be killed before brain measurement, which

20 precludes the possibility of getting longitudinal data on individual brain growth. Longitudinal

21 repeated measures would be beneficial to follow brain growth allocation over experimental

22 treatments, such as starvation/refeeding regimes and environmental manipulations, or during

23 ontogeny of different species or genotypes. For instance, some studies suggest that brain size can

24 be affected by addition of structures in fish rearing tanks (e.g. Kihslinger, Lema \& Nevitt, 2006;

25 Näslund et al., 2012), but such studies would benefit from following individuals through

26 ontogeny to be able to assess effects of differential growth patterns (Näslund \& Johnsson, 2014).

27 Non-invasive measurements, where the fish can be kept alive after brain measurements would

28 also be beneficial for brain size selection experiments (e.g. Kotrschal et al., 2013). One previous

29 study has measured brain size in live guppy Poecilia reticulata Peters, 1859 neonates, using a

30 method similar to the one presented here (the width of the optic tectum was measured from

31 photographs taken by a digital camera attached to a dissection microscope), but the method was

32 not validated (see supplemental information in Kotrschal et al., 2013). 
33 The aims of this paper are to (i) present a method for measuring gross brain size in small live fish

34 with semi-transparent heads, and (ii) compare the accuracy of measurements obtained from the 35 non-invasive method with invasive brain measurements. The species chosen for this study were 36 brown trout (in the fry stage) and zebrafish (in the young adult stage).

\section{MATERIALS AND METHODS}

\section{Subjects}

39 Wild brown trout Salmo trutta L. fry $(N=21$, size rage: $25.1-32.4 \mathrm{~mm}$ fork length) were 40 collected in the stream Norumsån, Sweden $\left(58^{\circ} 2.596^{\prime} \mathrm{N}, 11^{\circ} 50.760^{\prime} \mathrm{E}\right)$, using electrofishing (200$41300 \mathrm{~V}, \approx 1 \mathrm{~A}$, straight DC; L-600, LugAB, Luleå) in early June, 2013. After capture the trout were 42 kept in aquaria in the laboratory. Zebrafish Danio rerio (Hamilton, 1822$)(N=15$, size rage: 17.6 $43-22.9 \mathrm{~mm}$ standard length; adults) were bought from a pet shop and kept in aquaria. All fish 44 were subjected to behavioural experiments prior to brain-measurements, and were thus re-used as 45 experimental subjects. However, this fact should not influence the results of the present study. 46 Body length, head length and eye-diameter of the fish was measured from lateral photographs of 47 anaesthetised fish (precision $0.1 \mathrm{~mm}$ ), using ImageJ 1.46r (http://imagej.nih.gov/ij/). Fork length 48 was measured in brown trout, as this is the most common measure for salmonids, while standard 49 length was measured in zebrafish, due to the fact that some individuals had elongated caudal fins.

50 See figure 1 for definitions of the body, head and eye measurements.

\section{Non-invasive brain measurements}

52 A digital single lens reflex (DSLR) camera (Canon EOS 40D, Canon Inc., Tokyo) equipped with

53 a macro lens (Canon EF 100mm f/2.8 USM Macro 1:1) was vertically mounted on a copy-stand.

54 Underneath the camera, a LED light box (LightPad 920, Artograph Inc., Delano, MN) was placed 55 to provide light through the fish head, enhancing contrasts. A Petri dish was placed on the light 
56 box, underneath the lens, and filled with water containing a light dose of anaesthesia (2-

57 phenoxyethanol, 0.3-0.5 $\mathrm{ml} \cdot 1)$. Within the Petri dish a scale was placed as a reference (precision

$580.01 \mathrm{~mm})$. Fish were then placed in the Petri dish and photographed while being lightly

59 anaesthetized (i.e. resting at the bottom, still keeping equilibrium). For brown trout, one

60 photograph was normally taken, as these fish tend to rest in equilibrium (i.e. with the brain

61 perpendicular to the camera lens) on the bottom of the Petri dish. For zebrafish, which do not still

62 at the bottom of the dish, two to five photographs were taken, from which the best image (on

63 which the head was most symmetric) was selected for measurements. In a few cases, when the

64 fish was being too deeply anaesthetised before good photographs could be taken, I removed the

65 fish from the anaesthesia and photographed it at a later time point. The setup of the equipment is

66 illustrated in Fig. 2.

67 The brightness and contrast of the photographs were adjusted in Adobe Photoshop CS5.1 (Adobe

68 Systems Inc., San Jose, CA) to maximize visibility of the brain inside the head of the fish.

69 Measurements, i.e. the visible width, of telencephalon (Tel; only for brown trout), optic tectum

$70(\mathrm{TeO})$ and cerebellum $(\mathrm{Cb}$; only for brown trout) were taken from the digital photographs (see

71 Fig. 3) using ImageJ 1.46r. No non-invasive Tel- or Cb-measures were taken from zebrafish, as

72 these structures had relatively low visibility through the skin and skull in this species (Fig. 3c).

\section{Invasive brain measurements}

74 After being photographed the fish were killed by an overdose of 2-phenoxyethanol $(1 \mathrm{ml} \cdot 1)$, and

75 preserved in 4\% phosphate-buffered formaldehyde until dissection (10 months for trout, 1 week

76 for zebrafish). When dissected, the brains where cut off where the spinal cord enters the brain

77 stem and all nerves were cut as close to the brain as possible. The pituitary gland was removed

78 from all brains, as it was not successfully retained from all individuals when dissected.

79 Thereafter, they were photographed using a copy-stand mounted DSLR camera (Canon EOS 
80 40D), equipped with a super-macro lens (Canon MP-E 65mm f/2.8 1-5x Macro; set at ca. 1.5x

81 macro; Canon Inc.) and two external flash heads (Canon MT-24EX Macro Twin Lite Flash;

82 Canon Inc.). The camera was connected to a laptop computer, and pictures were captured from

83 the computer, using the live-view function of the DSLR and the EOS Utility 2.1 software (Canon

84 Inc.). The brains were put in Petri dishes which were filled with $0.01 \mathrm{M}$ phosphate buffer solution

85 to the height of the brain, using the liquid surface to align the brains horizontally. Within the Petri

86 dish a scale was placed as a reference (precision $0.01 \mathrm{~mm}$ ). After photography the brains were

87 blotted dry under light pressure from a finger and weighed (wet mass to the nearest $0.1 \mathrm{mg}$ ).

88 Measurements corresponding to the non-invasive measurements (see Fig. 4) were taken from the

89 digital photographs using ImageJ 1.46r. One zebrafish brain had the telencephalon and optic

90 tectum damaged during handling and no measurements of these structures could be taken from

91 the photographs; it was still possible to measure cerebellar width and mass of this brain.

\section{Analyses}

93 Body and brain measurements were analysed using Pearson correlation analyses in GraphPad

94 Prism 6 (GraphPad Software, Inc., La Jolla, CA). High correlation-factors would to a high degree

95 reflect the overall size of the fish; therefore, Pearson correlation analyses were also conducted

96 using the deviation from expected values as predicted by body length (i.e. the residuals from the

97 linear function of body length and dependent variables [brain measurements, head length and eye-

98 diameter]). The latter analyses show whether non-invasive and invasive measurements have

99 corresponding deviations from expected values, as predicted from body size. For the method to

100 be valid these correlations should be positive and statistically significant.

101 Brain mass was $\log _{\mathrm{e}}$-transformed prior to analysis as mass measurements scale allometrically to 102 length measurements. 
103 Animal ethics

104 The experiment was conducted in accordance with Swedish law and regulations and was

105 approved by the Ethical Committee on Animal Experiments in Gothenburg, Sweden. Brown trout

106 were kept under ethical license number 8-2011 and zebrafish under license number 274-2011.

107 RESULTS

108 Brown trout fry

109 As judged by the Pearson correlation coefficient, $r$, the non-invasive TeO-measure (Fig. 3b) 110 correlated well with the invasive measure of $\mathrm{TeO}$ width $(r=0.93$; Table 1, Fig. 5a) and brain 111 mass ( $\log _{\mathrm{e}}$ transformed) $(r=0.90$; Table 1, Fig. 5b). The non-invasive Tel- and Cb-measures did 112 not correlate as well with their corresponding brain areas, as measured from out-dissected brains 113 (Table 1). Eye-diameter correlated equally well with overall brain mass as the TeO-measure $(r=$ 114 0.90; Table 1; Fig. 5d), while head length did not ( $r=0.86$, Table 1, Fig. 5c). Body fork length 115 also correlated well with brain mass $(r=0.92$; Table 1$)$.

116 Pearson correlations of body-size-standardized variables (i.e. using the deviation from expected 117 values based on fork length) showed that non-invasive TeO-measurement corresponded well with 118 the invasive TeO-measurement $\left(r=0.75, \mathrm{p}<0.0001\right.$; Fig. 6a), and with the $\log _{\mathrm{e}}$-transformed 119 brain mass $(r=0.59, p=0.0047$; Fig. 6a). This shows that a fish with small TeO-width for its 120 body size, as measured on photographs of the living fish, also have smaller than expected TeO121 width, as measured from photographs of the out-dissected brain, and lower than expected brain 122 mass for its size. Similarly, fish with smaller than expected eyes also had lower than expected 123 brain mass, but the statistical evidence was weaker, albeit still significant $(r=0.48, p=0.026$;

124 Fig. 6a). Deviation from expected head length did not correlate significantly with deviation from 125 expected brain mass $(r=0.39, p=0.080)$, and comparisons of deviation from expectancy for 
126 non-invasive and invasive measurements of $\mathrm{Tel}$ and $\mathrm{Cb}$ did not produce significant correlations

127 either (Tel: $r=0.25, p=0.28$; Cb: $r=0.33, p=0.15$ ).

\section{Zebrafish}

129 The TeO-measure of zebrafish (Fig. 3d) proved to correlate well with optic tectum width 130 measured from invasive measurements $(r=0.92$; Table 1, Fig. 5a). Compared to brown trout fry,

131 the TeO-measure of zebrafish was less correlated with overall brain mass $\left(\log _{\mathrm{e}}\right.$ transformed $)(r=$ 132 0.79; Table 1, Fig. 4b). Head length (Fig. 5c), eye-diameter (Fig. 5d) and body standard length 133 had lower correlation with brain mass than the non-invasive TeO-measure $(0.60<r<0.72$; Table 134 1).

135 Correlations of body-size-standardized variables showed that non-invasive TeO-measurement 136 corresponded well with the invasive TeO-measurement ( $r=0.89, \mathrm{p}<0.0001$; Fig. 6b), and with 137 the $\log _{\mathrm{e}}$-transformed brain mass $(r=0.67, p=0.0078$; Fig. $6 \mathrm{~b})$. Like in the case of trout, this 138 shows that deviation from expected $\mathrm{TeO}$, as measured by the non-invasive and the invasive 139 methods, showed similar patterns. Deviation from expected eye-diameter and deviation from 140 expected $\log _{\mathrm{e}}$-transformed brain mass also correlated, but with lower statistical support $(r=0.52$, $141 p=0.043$; Fig. 6b). Deviation from expected head length did not correlate significantly with 142 deviation from expected brain mass $(r=0.44, p=0.098)$.

\section{DISCUSSION}

144 The results of this study show that a non-invasive measurement of the width of the optic tectum,

145 taken through the skull of a live fish, correlate well with the invasive measure of the same brain 146 structure. For brown trout fry the non-invasive TeO-measure also correlated well with brain wet 
147 mass. Other non-invasive measurements which could potentially be used as brain-size proxies

148 (eye-diameter and head length), were generally less good. Body length of the fish also predicted

149 brain size very well. However, experimental manipulations of growth rate (e.g. in studies

150 involving food restriction or selection/genetic modification for rapid somatic growth) may affect

151 the relation between body length and brain size, as fish with different growth rates have been

152 shown to differ in brain allometry (Pankhurst \& Montgomery, 1994; Devlin et al., 2012).

153 For both brown trout fry and zebrafish, the deviation from expected brain size based on body

154 length correlated well for non-invasive TeO-measurements and invasive TeO-measurments. The

155 correlations between non-invasive TeO-measurements and $\log _{\mathrm{e}}$-transformed brain mass, and

156 between eye-diameter and $\log _{\mathrm{e}}$-transformed brain mass were also significantly positive, with the

157 former having higher precision than the latter for both species. Corresponding comparisons for

158 head size vs. $\log _{\mathrm{e}}$-transformed brain mass were not satisfactory (i.e. non-significant).

159 The correlations between absolute values of non-invasive and invasive measurements were in

160 general a bit lower for zebrafish than for brown trout fry. The zebrafish were smaller than the

161 trout fry, but had less transparent heads, which may explain part of the lower correlation factor.

162 However, body measurements (standard length, eye-diameter and head length) also showed lower

163 correlations in zebrafish than in brown trout. This indicates that the zebrafish has more individual

164 variation in body and/or brain morphometrics than brown trout. It should be pointed out that the

165 zebrafish were fixed in formaldehyde for a substantially shorter period than the trout, which

166 could have contributed to variation in invasive brain measurements. The initial period of fixation

167 is generally when the most changes are occurring in brain tissue (Weisbecker, 2012; Ridgway \&

168 Hanson, 2014).

169 The main advantage of the non-invasive TeO-measure is that it requires substantially less 170 preparation and handling time than invasive methods, making large sample sizes feasible. If there 171 is great among-individual variation in brain size in experimental groups, a large sample size of 
172 decent brain measures could be better than a few really good brain measures. The TeO-measure

173 also makes it possible to repeatedly measure brain growth in small live fish, e.g. through

174 ontogeny or in growth-manipulation experiments. The main disadvantages are that the $\mathrm{TeO}-$

175 measure is limited to estimating optic tectum width and whole brain size, and it only works as

176 long as the brain is visible through the head. The latter fact restricts repeated measures into

177 adulthood in many species where pigmentation and thickness of tissue and bone above the brain

178 limits the visibility of the brain.

179 The TeO-measure could likely be useful for estimating brain size in many small fish species and

180 juvenile stages of larger fish species. However, since the accuracy of the TeO-measure may differ

181 among species, validation is needed before the method is applied on a new species.

\section{General advice}

183 When taking photographs, it is important to make sure that the reference scale is at the same

184 height as the dorsal-most part of the fish head in the Petri dish; otherwise it will be out of focus 185 on the images.

186 Dosage of anaesthesia was more critical for zebrafish than for trout. Trout have open 187 (physostome) swim bladder (Fänge, 1983), which is quickly emptied, and large pectoral fins 188 which they use as support when resting at the bottom of the Petri dish. Consequently, the fish is 189 relatively stable through the sedation. Zebrafish have a closed (physoclist) swim bladder (Fänge, 190 1983) and only use their relatively small pelvic fins as support while resting at the bottom. This 191 makes the zebrafish more sensitive to changes in their equilibrium during anaesthesia, and they 192 can flip over completely relatively quickly even if not fully anaesthetized. After a fish has lost 193 equilibrium and flipped over, it needs to recover to regain equilibrium and then be re194 anaesthetized. For this reason, the concentration of anaesthesia needs to be more carefully chosen 195 for species with closed swim bladder, as compared to species with open or no swim bladder. The 
196 efficiency of anaesthesia depends largely on the type of anaesthesia used, species and water

197 temperature (Ross \& Ross, 2008), and suitable dosage should be tested out for each experiment.

198 In general, the method described works only on fish where the brain is visible through the skin

199 and skull. The more transparent the head is, the better the measurements. This makes the method

200 suitable, primarily, for young fish. Some adult fish of small size, like zebrafish, can also be

201 measured using the method. Most likely, there are size thresholds when the method stops working 202 for both zebrafish and brown trout, and likely for most other candidate species. At some 203 particular size, the visibility through the skin and skull will be obscured by the pigmentation of 204 the skin and/or the thickness of the skull. Based on personal observations, some fish species 205 seem unsuitable for the method, despite small size (e.g. fish with steep foreheads like some 206 species of cichlids), while other species have good head transparency also as full grown adults 207 (e.g. guppies).

208 To make the brain maximally visible through the semi-transparent head, it is advisable to keep 209 the fish in a bright environment for a while prior to the photography, to make the coloration of the 210 head lighter, and the outline of the brain more easily detected.

211 Caution should be taken when analysing fish of different ontogenetic stages, as the relative size 212 of the optic tectum, as compared to other brain structures, may change during ontogeny (Näslund 213 et al., 2012).

\section{ACKNOWLEDGEMENTS}

215 Per Saarinen is thanked for his assistance in collecting, caring for and photographing the trout fry. 216 Joachim Sturve and Britt Sjöqvist are thanked for caring for the zebrafish. Jörgen I. Johnsson and 217 three reviewers provided valuable comments to earlier versions the manuscript. 
REFERENCES

219 Burns JG, Rodd FH. 2008. Hastiness, brain size and predation regime affect the performance of

220 wild guppies in a spatial memory task. Animal Behaviour 76:911-922 DOI

$221 \quad$ 10.1016/j.anbehav.2008.02.017

222 Devlin RH, Vandersteen WE, Uh M, Stevens ED. 2012. Genetically modified growth rates 223 affects allometry of eye and brain in salmonids. Canadian Journal of Zoology 90:193224 DOI $10.1139 / Z 11-126$

225 Ebbesson LOE, Braithwaite VA. 2012. Environmental effects on fish neural plasticity. Journal 226 of Fish Biology 81:2151-2174 DOI 10.1111/j.1095-8649.2012.03486.x

227 Fraser TWK, Fjelldal PG, Skjæraasen JE, Hansen T, Mayer I. 2012. Triploidy alters brain 228 morphology in pre-smolt Atlantic salmon Salmo salar: possible implications for behaviour. Journal of Fish Biology 81:2199-2212 DOI 10.1111/j.10958649.2012.03479.x Pharmacology 97:111-158 DOI 10.1007/BFb0035347

Gonda A, Herczeg G, Merilä J. 2013. Evolutionary ecology of intraspecific brain size variation: a review. Ecology and Evolution 3:2751-2764 DOI 10.1002/ece3.627

235 Marchetti MP, Nevitt GA. 2003. Effects of hatchery rearing on brain structures of rainbow trout, Oncorhynchus mykiss. Environmental Biology of Fishes 66:9-14 DOI 10.1023/A:1023269221678

238 Kihslinger RL, Lema SC, Nevitt GA. 2006. Environmental rearing conditions produce 239 forebrain differences in wild chinook salmon Oncorhynchus tshawytscha. Comparative Biochemistry and Physiology, Part A: Molecular \& Integrative Physiology 145:145-151 DOI 10.1016/j.cbpa.2006.06.041 
242 Kotrschal K, Palzenberger M. 1992. Neuroecology of cyprinids: comparative, quantitative histology reveals diverse brain patterns. Environmental Biology of Fishes 33:135-152

Näslund J, Johnsson JI. 2014. Environmental enrichment for fish in captive environments: effects of physical structures and substrates. Fish and Fisheries In Press DOI 10.1111/faf.12088

Näslund J, Aarestrup K, Thomassen ST, Johnsson JI. 2012. Early enrichment effects on brain development in hatchery-reared Atlantic salmon (Salmo salar): no evidence for a critical period. Canadian Journal of Fisheries and Aquatic Sciences 69:1481-1490 DOI 10.1139/F2012-074

Pankhurst NW, Montgomery JC. 1994. Uncoupling of visual and somatic growth in the rainbow trout Oncorhynchus mykiss. Brain, Behavior and Evolution 44:149-155 DOI $10.1159 / 000113586$

Park PJ, Bell MA. 2010. Variation of telencephalon morphology of the threespine stickleback (Gasterosteus aculeatus) in relation to inferred ecology. Journal of Evolutionary Biology 
265 Park PJ, Chase I, Bell MA. 2012. Phenotypic plasticity of the threespine stickleback Gasterosteus aculeatus telencephalon in response to experience in captivity. Current Zoology 58:189-210

268

269

270

271

272

273

274

275

276

277

278

279

280

281

282

283

284
Pollen AA, Dobberfuhl AP, Scace J, Igulu MM, Renn SCP, Shumway CA, Hofmann HA. 2007. Environmental complexity and social organization sculpt the brain in Lake Tanganyikan cichlid fish. Brain, Behavior and Evolution 70:21-39 DOI $10.1159 / 000101067$

Ridgway SH, Hanson AC. 2014. Sperm whales and killer whales with the largest brains of all toothed whales show extreme differences in cerebellum. Brain, Behavior and Evolution 83:266-274 DOI 10.1159/000360519

Ross LG, Ross B. 2008. Anaesthetic and sedative techniques for fish, $3^{\text {rd }}$ edition. WileyBlackwell.

Ullmann JFP, Cowin G, Collin SP. 2010. Quantitative assessment of brain volumes in fish: comparison of methodologies. Brain, Behavior and Evolution 76:261-270 DOI $10.1159 / 000321467$

Ullmann JFP, Cowin G, Kurniawan ND, Collin SP. 2010. A three-dimensional digital atlas of the zebrafish brain. NeuroImage 51:76-82 DOI 10.1016/j.neuroimage.2010.01.086

Weisbecker V. 2012. Distortion in formalin-fixed brains: using geometric morphometrics to quantify the worst-case scenario in mice. Brain Structure \& Function 217:677-685 DOI 10.1007/s00429-011-0366-1 


\section{Table 1 (on next page)}

Table 1

Correlations between different measurements of fish brains and parameters for ordinary least squares regression lines, with $95 \%$ confidence intervals for each parameter given within brackets. 


\begin{tabular}{|c|c|c|c|}
\hline & $\begin{array}{l}\text { Correlation } \\
\text { coefficient }(r)\end{array}$ & Slope & Y-intercept \\
\hline \multicolumn{4}{|l|}{ Brown trout fry } \\
\hline TeO-measure vs. Optic tectum width & $0.93(0.84-0.97)$ & $0.87(0.71-1.03)$ & $0.41(-0.01-0.83)$ \\
\hline TeO-measure vs. $\log _{\mathrm{e}}$ (Braiß)mass) & $0.90(0.76-0.96)$ & $0.99(0.75-1.22)$ & $-7.49(-8.08--6.89)$ \\
\hline Tel-measure vs. Telencepha苂n width & $0.57(0.18-0.80)$ & $0.64(0.19-1.08)$ & $0.47(0.00-0.93)$ \\
\hline Cb-measure vs. Cerebellum & $0.63(0.27-0.83)$ & $0.56(0.22-0.87)$ & $0.48(0.12-0.85)$ \\
\hline Eye-diameter vs. $\log _{\mathrm{e}}$ (Braira mass) & $0.90(0.77-0.96)$ & $1.32(1.02-1.62)$ & $-7.49(-8.05--6.90)$ \\
\hline Head length vs. $\log _{e}$ (Brain mass) & $0.86(0.69-0.94)$ & $0.28(0.20-0.36)$ & $-6.75(-7.26--6.24)$ \\
\hline $\begin{array}{l}\text { Body length vs. } \log _{\mathrm{e}} \text { (Braif mass) } \\
\text { Zebrafish }\end{array}$ & $0.92(0.82-0.97)$ & $0.08(0.06-0.09)$ & $-7.20(-7.64--6.75)$ \\
\hline TeO-measure vs. Optic tectum width & $0.92(0.75-0.97)$ & $0.71(0.52-0.90)$ & $0.61(0.18-1.05)$ \\
\hline TeO-measure vs. $\log _{\mathrm{e}}$ (Brain mass) & $0.79(0.45-0.93)$ & $1.64(0.84-2.44)$ & $-9.35(-11.16-7.54)$ \\
\hline Eye-diameter vs. $\log _{\mathrm{e}}$ (Brain mass) & $0.72(0.33-0.90)$ & $1.31(0.55-2.07)$ & $-8.00(-9.37--6.63)$ \\
\hline Head length vs. $\log _{\mathrm{e}}$ (Brain mass) & $0.70(0.30-0.89)$ & $0.38(0.15-0.60)$ & $-7.23(-8.20--6.26)$ \\
\hline Body length vs. $\log _{e}($ Brain mass $)$ & $0.60(0.14-0.85)$ & $0.06(0.01-0.11)$ & $-6.93(-7.94--5.92)$ \\
\hline
\end{tabular}




\section{Figure 1}

Figure 1

Body measurements illustrated on a schematic zebrafish. Fork length (used for brown trout): from the tip of the snout to the fork of the caudal fin (i.e. the end of the central caudal fin ray); standard length (used for zebrafish): from the tip of the snout to the end of the spine; head length: from the tip of the snout to the dorsal-most end of the gill opening; eye-diameter: dorso-lateral diameter of the eye cavity.

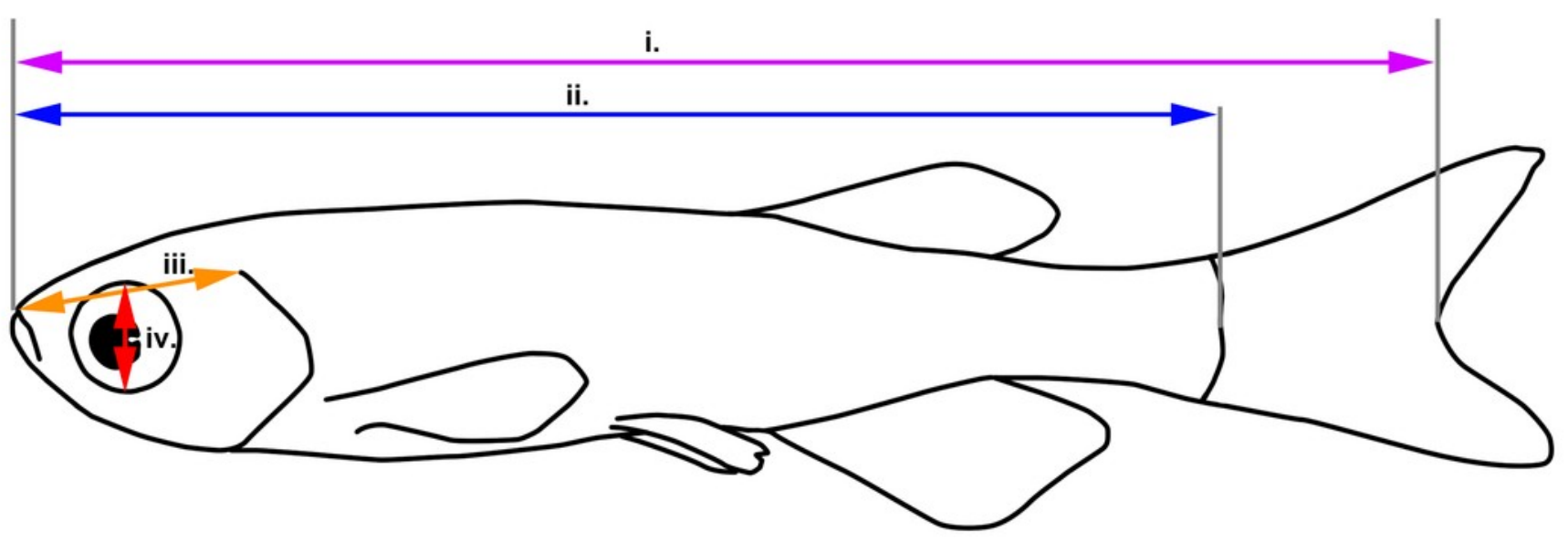

i. Fork length iii. Head length

ii. Standard length iv. Eye diameter 


\section{Figure 2}

Figure 2

The brain photography setup. The DSLR camera (1.) was mounted vertically on a vertical copy-stand (2.) equipped with a sled which could be adjusted vertically (3.). Underneath the lens of the camera, on a light box (4.), a Petri dish was placed, containing water-dissolved anaesthesia and the fish.

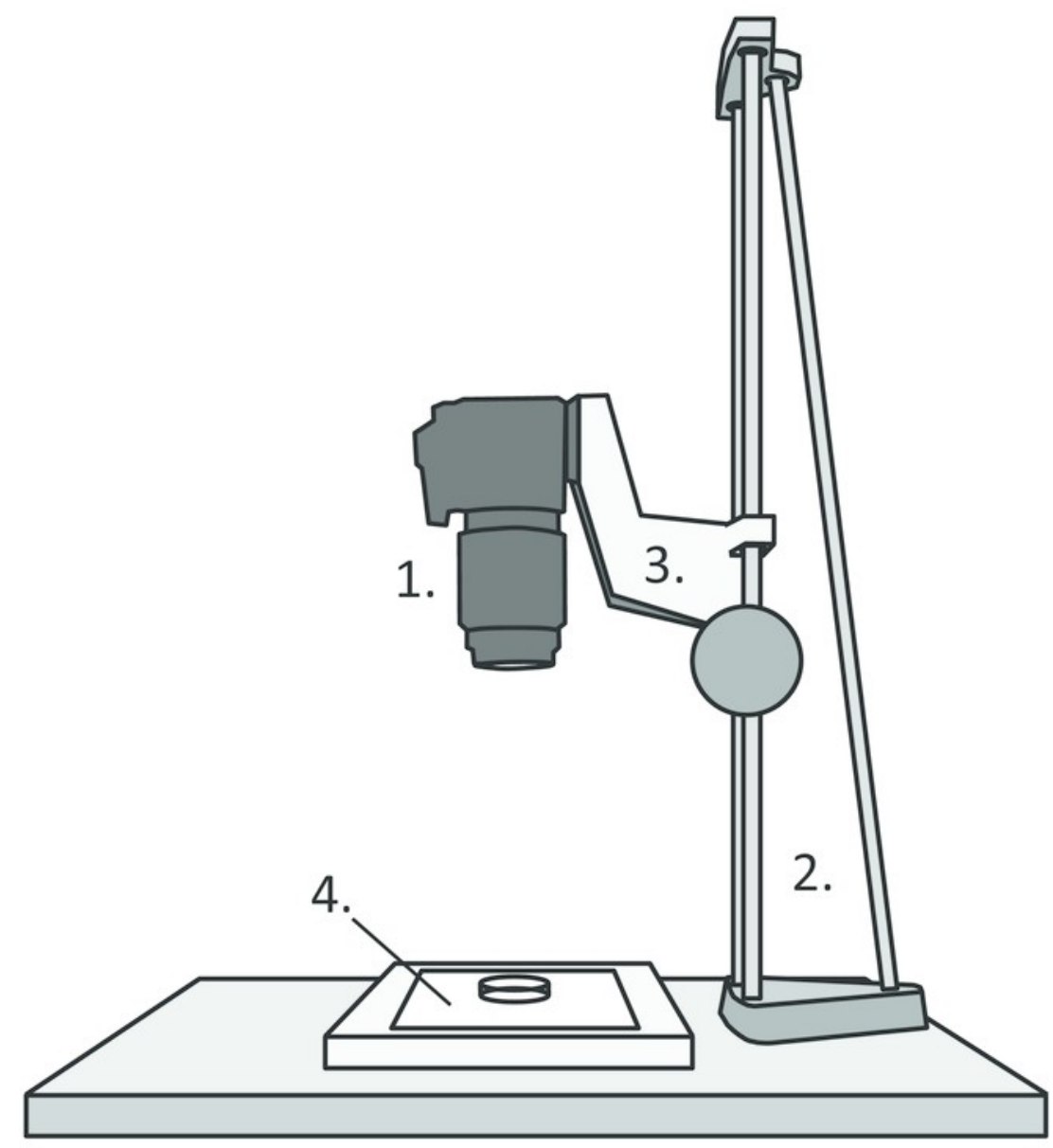




\section{Figure 3}

Figure 3

Dorsal photographs of brown trout fry (fork length: $31.1 \mathrm{~mm})(A, B)$, and young adult zebrafish (fork length: $23.3 \mathrm{~mm})(C, D)$. B and D show measurements taken from images.

See Fig. 3 for photos of out-dissected brains. Abbreviations: Tel = telencephalon; $\mathrm{TeO}=$ optic tectum; $\mathrm{Cb}=$ cerebellum.

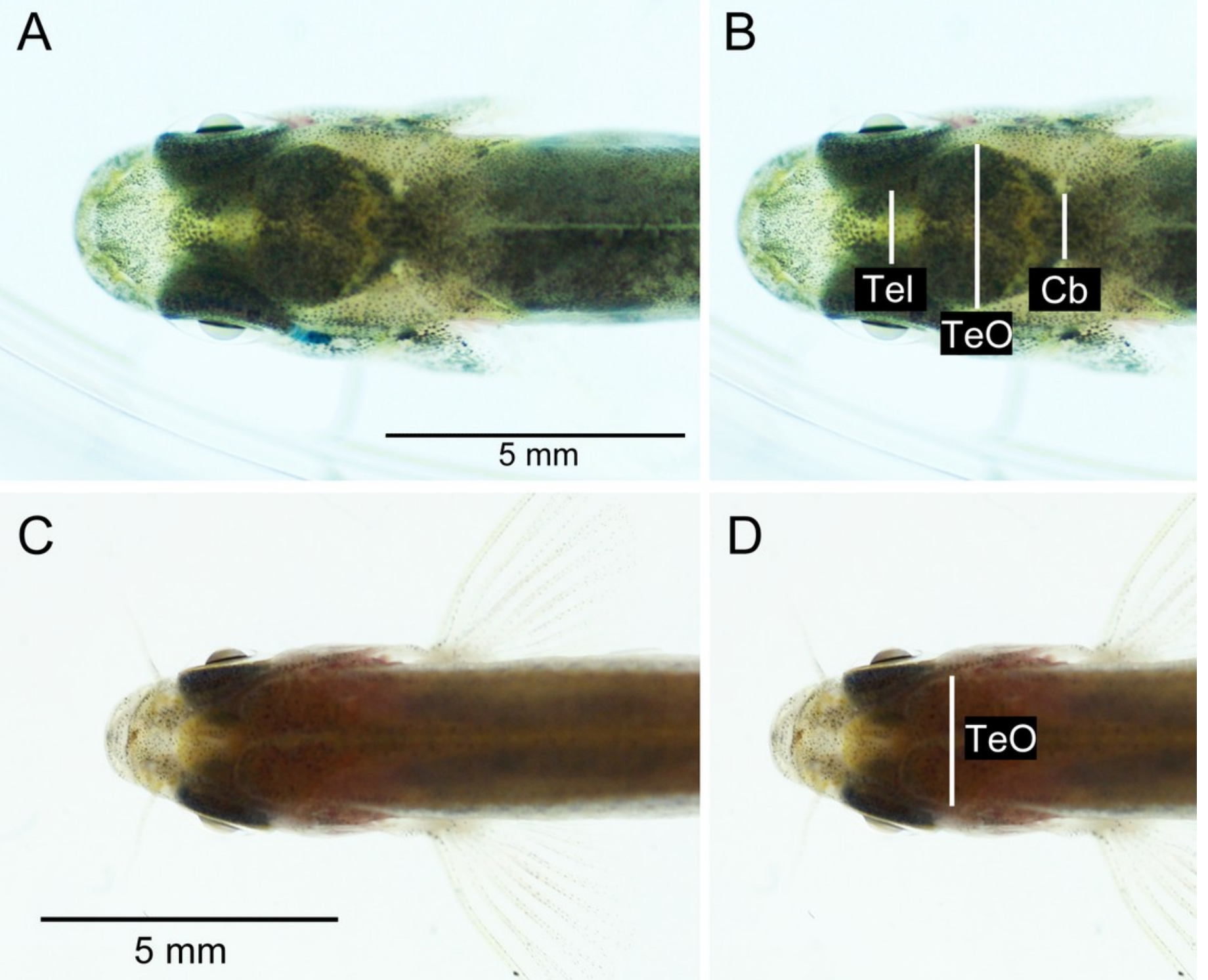




\section{Figure 4}

Figure 4

Dorsal photographs of brown trout fry brain $(A)$, and juvenile zebrafish brain $(C)$, with measurements taken marked as white lines. B and D show outlines of the trout brain and the zebrafish brain, respectively. Abbreviations: $\mathrm{Tel}=$ telencephalon; $\mathrm{TeO}=$ optic tectum; $\mathrm{Cb}=$ cerebellum. Anterior parts of the brains are pointing to the left.
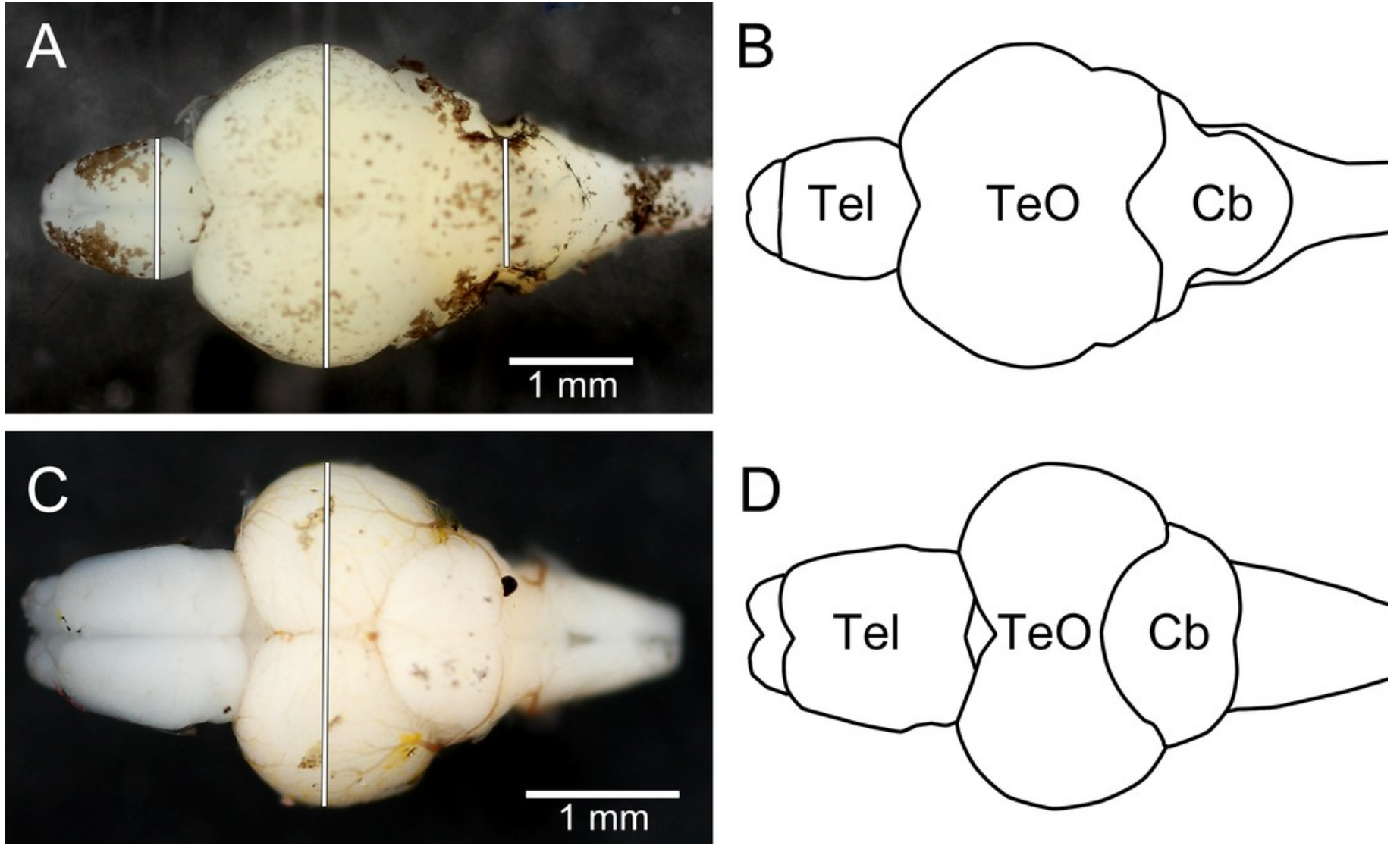


\section{Figure 5}

Figure 5

Correlations between non-invasive measurements ( $\mathrm{X}$-axes) and invasive brain measurements (Y-axes). Brown trout fry: black; zebrafish: red. Pearson r-values and parameters for the regression lines are presented in Table 1. 

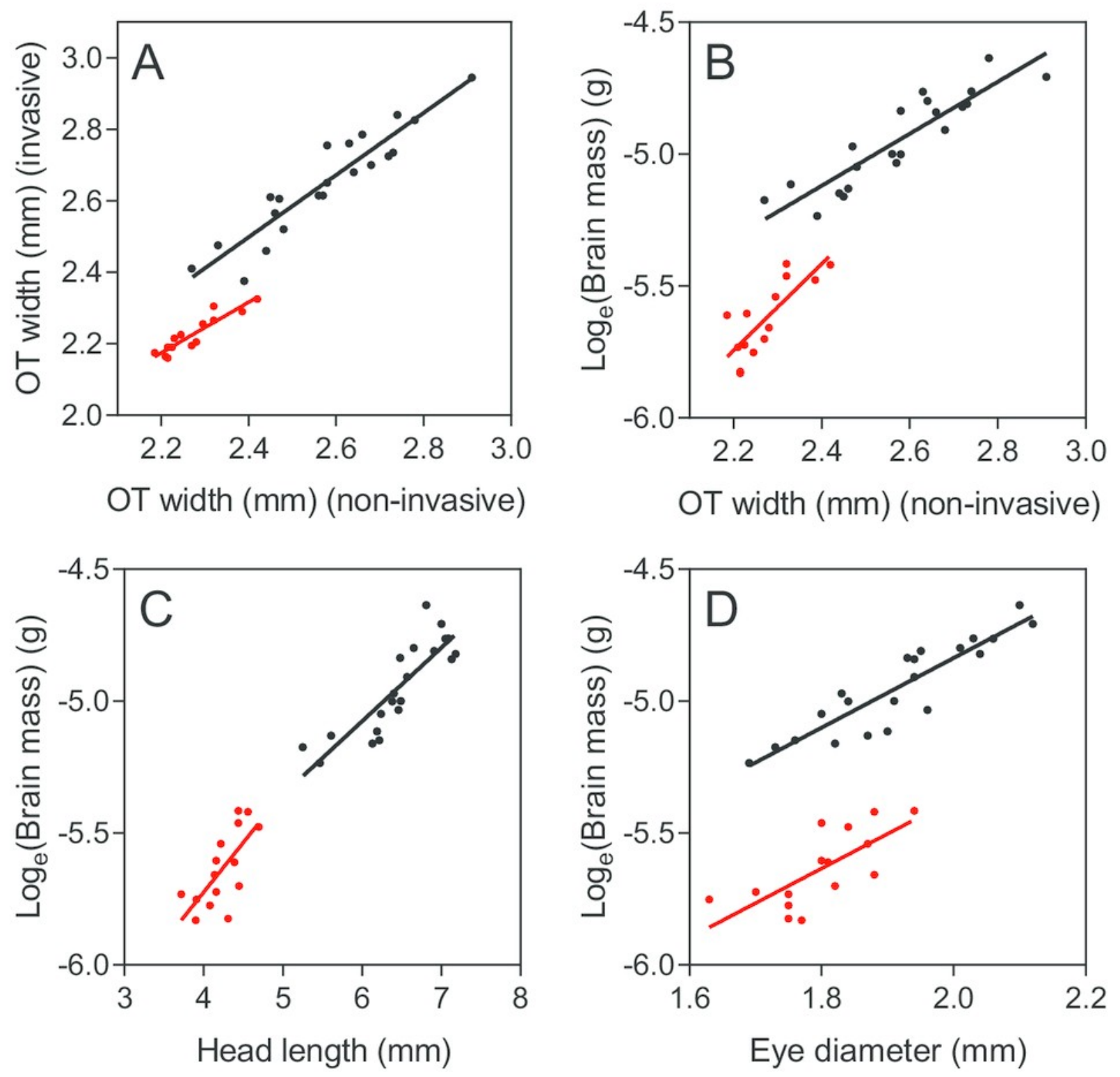


\section{Figure 6}

Figure 6

Correlations between non-invasive measurements ( $\mathrm{X}$-axes) and invasive brain measurements (Y-axes), standardized for body size. A: brown trout fry; B: zebrafish. Dotted lines show 95\% confidence intervals of the regression lines.

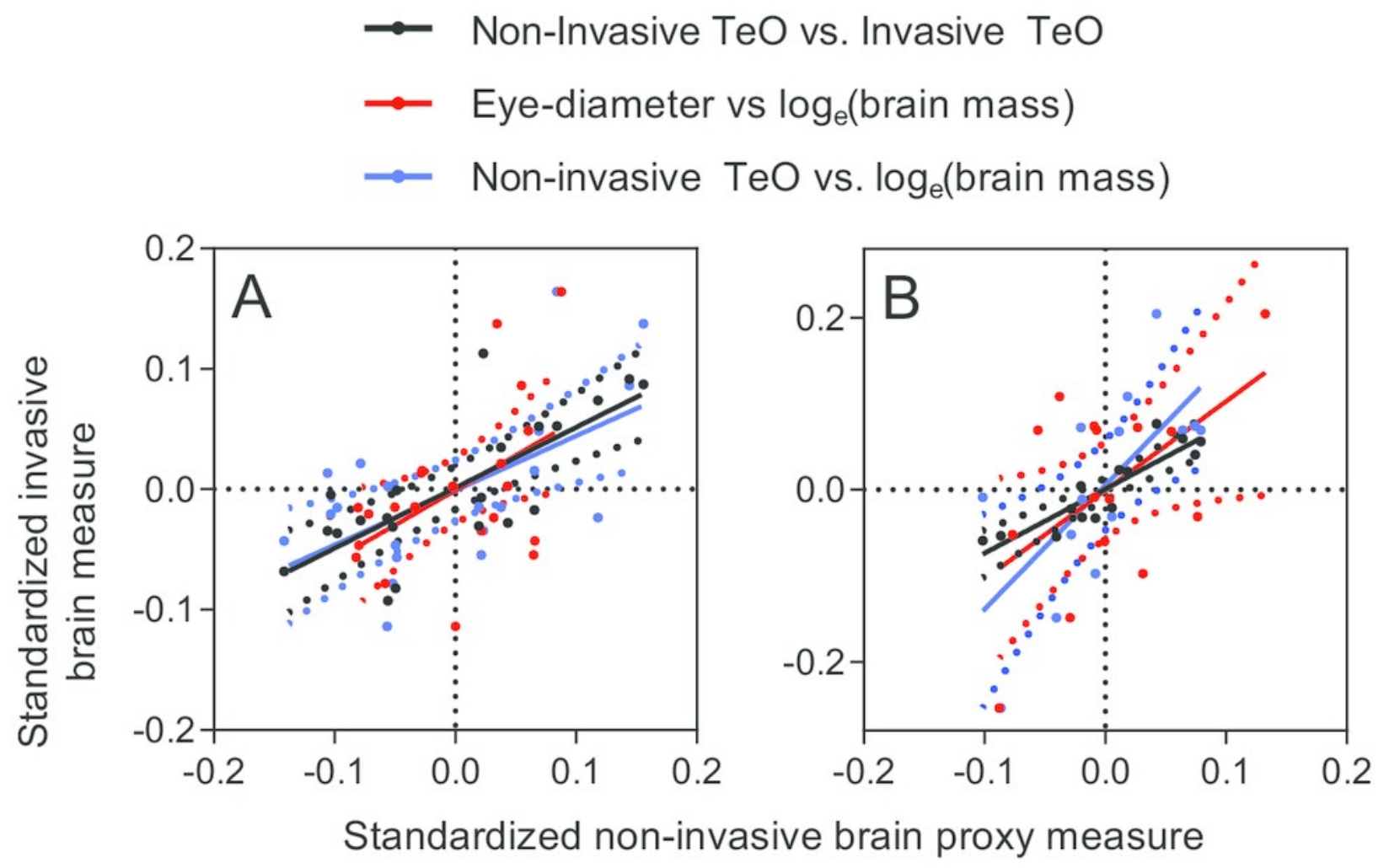

